\title{
Stability markers in control theory
}

\author{
Egor Polilov ${ }^{1, *}$ and Olga Batiushina ${ }^{2}$ \\ ${ }^{1}$ Donbass State Technical University, Department of Automation and Electrical Engineering Systems, Ukraine \\ ${ }^{2}$ Donbass State Technical University, Department of Management, Ukraine
}

Abstract. The linear dynamic systems stability criterion, allowing one to identify the defective/failed coefficients in the characteristic polynomial at each time quantum, is presented for the first time.

\section{Introduction}

Is there a great probability to generate Hurwitz's polynomial in a random way? And, fundamentally, is it possible to achieve Hurwitz's features with a selection $(n+1)$ pieces of $\sigma_{j}, \forall j=\overline{0, n}$ for the high order systems? It is appropriate to concentrate the answer to such questions on the extent/volume assessing of the two clusters, stability and instability, as the interaction of extreme opposites.

It should be admitted that the potential of the socalled stability criteria [1, 2] by Hermite-Biller's theorem about the radicals alternation, Sturm's method for the rational function index calculation, Routh's algorithm, Hurwitz and Lienard-Schipar's determinantal conditions, quadratic forms based on Schur's method, Chebotarev's theorem, a generalization for the entire functions of Hermite-Biler's theorem, Pontryagin's theorem on stable quasipolynomials, and others has been exhausted for formulated problems solving and it is quite insignificant despite of the apparent theoretical harmony and algorithmic/computational clutters. Is it necessary to confirm the stability or instability dynamical systems? Out-of-date. With the same success, the step response $h(t)$ step(sys) is the essence of the answer to this question, in addition geometrically. And a map of the radicals pzmap(sys) distribution is more informative (step(sys) and pzmap(sys) the computational procedures of Control System Toolbox in MATLAB). But, in fact, besides the ascertaining of the instability one should clearly understand what to do with non Hurwitz's polynomial? When a patient needs emergency medical care, it is necessary to provide it qualifiedly, but not contemplating the obvious fact to convince yourself that it is really required.

In $[3,4]$ authors propose a method for computing the distance of a stable polynomial to the set of unstable ones (both in the Hurwitz and in the Schur case). The method is based on the reformulation of the problem as the structured distance to instability of a companion matrix associated to a polynomial.
The ideology of stability markers, which at each time quantum $t_{k}$ visually identifies the defective/failed $\sigma_{k}(t), \forall k \in \overline{0, n}$ from a set of the coefficients $\sigma_{j}(t), \forall j=\overline{0, n}$ of the nonstationary algebraic equation:

$G(p, t)=\sigma_{n}(t) p^{n}+\sigma_{n-1}(t) p^{n-1}+\ldots+\sigma_{0}(t)=0$,

and points the recovery ways of each coefficient $\sigma_{k}(t), \forall k \in \overline{0, n}$, so it is the important issue. There are no similar solutions in the automatic control theory.

\section{Material and researches results}

Definition. There is the $2 \times n$ pieces set spectrum of the geometric averages $\Omega_{k}(t): \underset{j=1, n-k}{\operatorname{gmean}}\left(c_{j}(t)\right), \forall k \in \overline{0, n}$ locations of the instant dynamic solutions $\left\langle c_{1}(t), c_{2}(t)\right.$, $\left.\ldots, c_{n-k}(t)\right\rangle_{k}, \forall k \in \overline{0, n}$, fig. 1 , formed with a serial dropping of the polynomial (1) highest (2) and youngest (3) degrees. New solutions of the so-called enclosed polynomials of $(n-i, j)$ orders define other combinations of valid and complex conjugated radicals on the complex plane. In their turn, these radicals are geometrically averaged with the own "universe brick" $R_{i(k)}(t)=\sqrt[n-i]{\left|\sigma_{0}(t) / \sigma_{n-i}(t)\right|} \forall k \in \overline{0, n}$ of the encloses on the right:

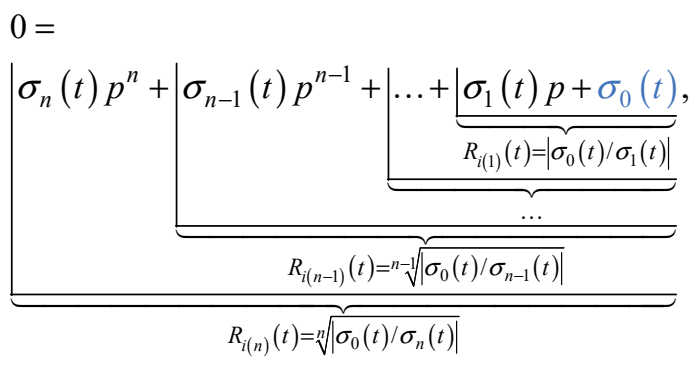

\footnotetext{
* Corresponding author: egor.polilov@gmail.com
} 
and $\quad R_{i(k)}^{*}(t)=\sqrt[n-j]{\left|\sigma_{n-j}(t) / \sigma_{n}(t)\right|}, \forall j \in \overline{0, n}$ of the encloses on the left:

$0=$

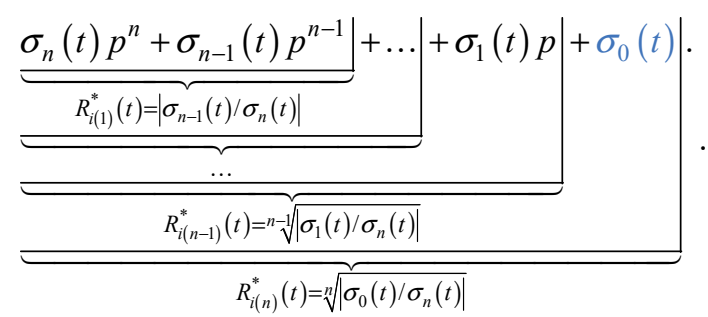

Here and further $R_{i(k)}$ and $R_{i(k)}^{*}$ are the radii of Polilov's- Motchenko's invariants [5, 6].
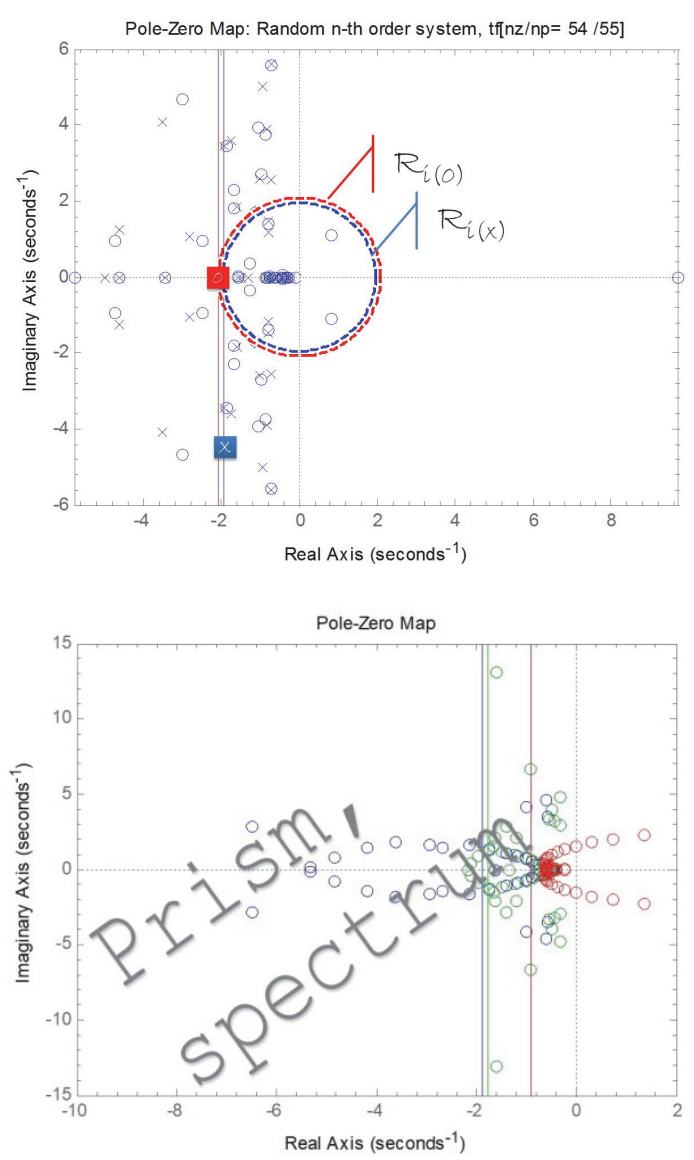

Fig. 1. A distribution map of radicals, geometric averages $R_{i(0)}$ and $R_{i(\times)}$ and spectrum $\Omega_{k} \forall k \in \overline{0,2 \times n}$ of the characteristic polynomial (verticals, 3 of them are shown).

The horizontal and vertical numbering of the $\mathbf{P}(t)$ matrix (4) cells will be associated with the degrees of the polynomial (1), beginning from the highest left top one $\sigma_{n-i}(t) p^{n-i} \quad$ and $\quad \sigma_{n-j}(t) p^{n-j} \quad$ for $\quad i, j=\overline{0, n}$, accordingly, thereby the localization of each cell $\mathbf{P}_{i j}$ will be attached to the spectrum of the geometric averages:

$$
\begin{aligned}
& \mathbf{P}_{G(p, t)}=
\end{aligned}
$$

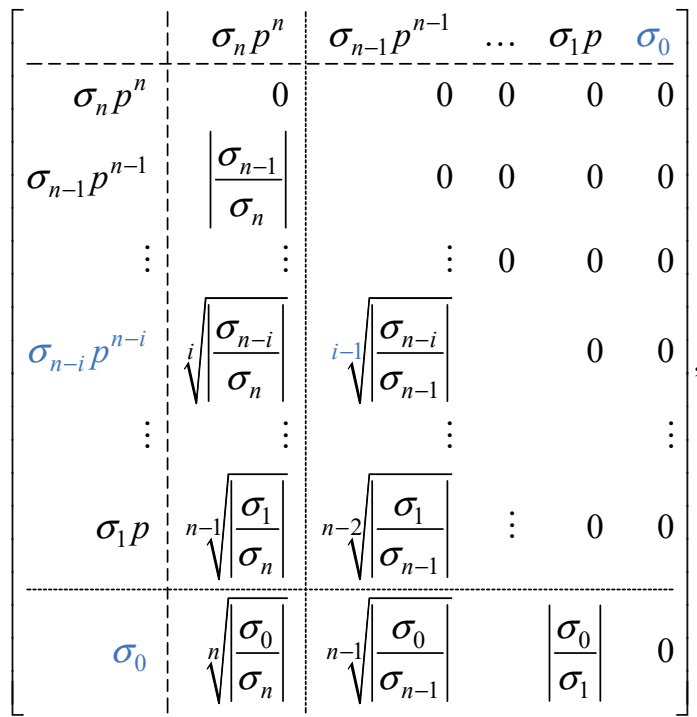

$\sigma_{j}(t), \forall j=\overline{0, n}$ are still non-stationary in the text here and further, time dependence symbol is omitted because of the matrix overcharging.

The first column and the last row of $\mathbf{P}$-matrices are those investments on the right (2) and on the left (3), which are separated by a dotted line. The other elements $\mathbf{P}_{i j}$ should be associated with the so-called hybrid investments, mentally leaving any adjacent components with $k$ degree in the polynomial $\left(\sigma_{m-j}(t) p^{m-j} \ldots \sigma_{i}(t) p^{i}\right)$ during the deliverance from the part of the $j=\overline{1, m}$ oldest and the $i=\overline{0, j}$ youngest degrees $p$ at the same time.

Definition. The linear non-stationary dynamic system is characterized by $m(m-1) / 2$ numbers of the geometric averages $\Omega_{k}(t), \quad \forall k=\overline{0, m}$ of the polynomial numerator $A(p, t)$ zeros and by $n(n-1) / 2$ numbers of the geometric averages $\Omega_{k}(t), \forall k=\overline{0, n}$ of the selected transfer function polynomial denominator $B(p, t)$ poles

$$
\begin{aligned}
& W(p, t)=\frac{A(p, t)}{B(p, t)}=k_{0} \frac{\sum_{i=0}^{m} \alpha_{m-i}(t) p^{m-i}}{\sum_{j=0}^{n} \beta_{n-j}(t) p^{n-j}}= \\
& =k_{0} \frac{\alpha_{m}(t) p^{m}+\alpha_{m-1}(t) p^{m-1}+\ldots+\alpha_{0}(t)}{\beta_{n}(t) p^{n}+\beta_{n-1}(t) p^{n-1}+\ldots+\beta_{0}(t)},
\end{aligned}
$$

forming two lower triangular $\mathbf{P}$ matrices, $(m+1,1)$ and $(n+1,1)$ cells of $\mathbf{P}$ matrices are nothing less than the geometric average $\Omega_{k}(t): \underset{j=\overline{1, n-k}}{\text { gmean }}\left(c_{j}(t)\right), \forall k \in \overline{0, n}$ of 
the instant dynamic solutions $\left\langle c_{1}(t), c_{2}(t), \ldots\right.$, $\left.c_{n-k}(t)\right\rangle_{k}$ location, $\forall k \in \overline{0, n}$ encloses of the numerator $A(p, t)$ polynomial and $\forall k \in \overline{0, n}$ attachments of the denominator $B(p, t)$ polynomial:

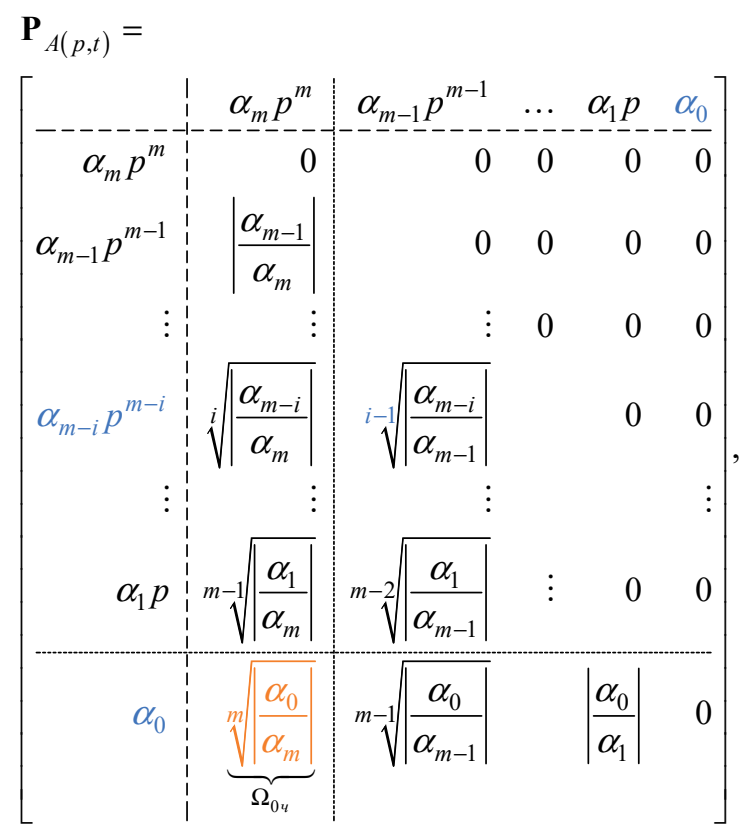

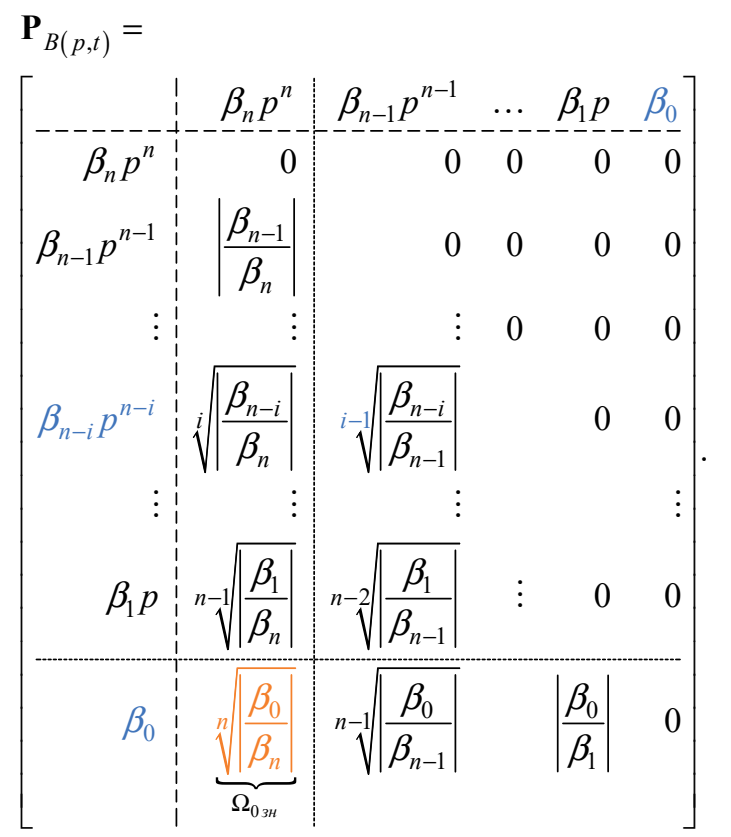

In other words, the scaling constants of cells $\mathbf{P}_{i j}=\sqrt[i-j]{\left|\alpha_{m-i}^{*} / \alpha_{m-j}^{*}\right|}$ of $\mathbf{P}$-matrices define the measure of the dominance $\mathbf{P}_{i j}>1$ or of the subordination $\mathbf{P}_{i j}<1$ of any enclose from $\left(\alpha_{m-j}(t) p^{m-j} \ldots \alpha_{i}(t) p^{i}\right)$ to the invariant $\left(\alpha_{m}(t) p^{m}+\alpha_{0}(t)\right)=0$.

The dimension of the subdiagonal cells of $\mathbf{P}$ matrices $[\mathrm{rad} / \mathrm{sec}]$, all cells of $\mathbf{P}$-matrices (6), (7) are the frequencies, that can be seen obviously, if we present the transfer function (5) with the normalized polynomials $p_{\Omega_{(0)}}=p / \Omega_{(0)}$ and $p_{\Omega_{(\times)}}=p / \Omega_{(\times)}$:

$$
\begin{aligned}
& W\left(p_{\Omega_{(0, x)}}, t\right)=\frac{A\left(p_{\Omega_{(0)}}, t\right)}{B\left(p_{\Omega_{(\times)}}, t\right)}=k_{0} \frac{\sum_{i=0}^{m} \alpha_{m-i}^{*}(t) p_{\Omega_{(0)}^{m-i}}^{m} \beta_{j=0}^{*}(t) p_{\Omega_{(\times)}^{n-j}}^{n}}{\sum_{n-j}^{n-1}+\ldots+\alpha_{0}(t)} \\
& =k_{0} \frac{\alpha_{m}^{*}(t) p_{\Omega_{(0)}^{m}}^{m}+\alpha_{m-1}^{*}(t) p_{\Omega_{(0)}}^{m-1}}{\beta_{n}^{*}(t) p_{\Omega_{(\times)}}^{n}+\beta_{n-1}^{*}(t) p_{\Omega_{(\times)}}^{n-1}+\ldots+\beta_{0}(t)},
\end{aligned}
$$

then, for example, the polynomial $\left(\alpha_{m-1}(t) p^{m-1} \ldots \alpha_{1}(t) p\right)$ of the hybrid enclose first iteration in $A(p, t)$ determines the corresponding cell of the $\mathbf{P}$-matrix (6): $\sqrt[m-2]{\left|\frac{\alpha_{1}}{\alpha_{m-1}}\right|}=\sqrt[m-2]{\left|\frac{\left(\alpha_{1}^{*} / \Omega_{(0)}\right)}{\left(\alpha_{m-1}^{*} / \Omega_{(0)}^{m-1}\right)}\right|}=$ $\underbrace{\sqrt[m-2]{\left|\alpha_{1}^{*} / \alpha_{m-1}^{*}\right|}}_{\text {const },[\text { relative units }]} \times \underbrace{\Omega_{(0)}[\mathrm{rad} / \mathrm{sec}]}_{\text {geometrical mean }}$ etc., and (6) is appropriate to present in the form:

$$
\begin{aligned}
& \mathbf{P}_{A(p, t)}=\mathbf{P}_{A(p, t)}^{*}[\text { relative units }] \times \Omega_{(0)}[\mathrm{rad} / \mathrm{sec}]= \\
& =\mathbf{P}_{A(p, t)}^{*} \times \sqrt[m]{\left|\alpha_{0} / \alpha_{m}\right|},
\end{aligned}
$$

$$
\begin{aligned}
& \mathbf{P}_{A(p, t)}^{*}=
\end{aligned}
$$

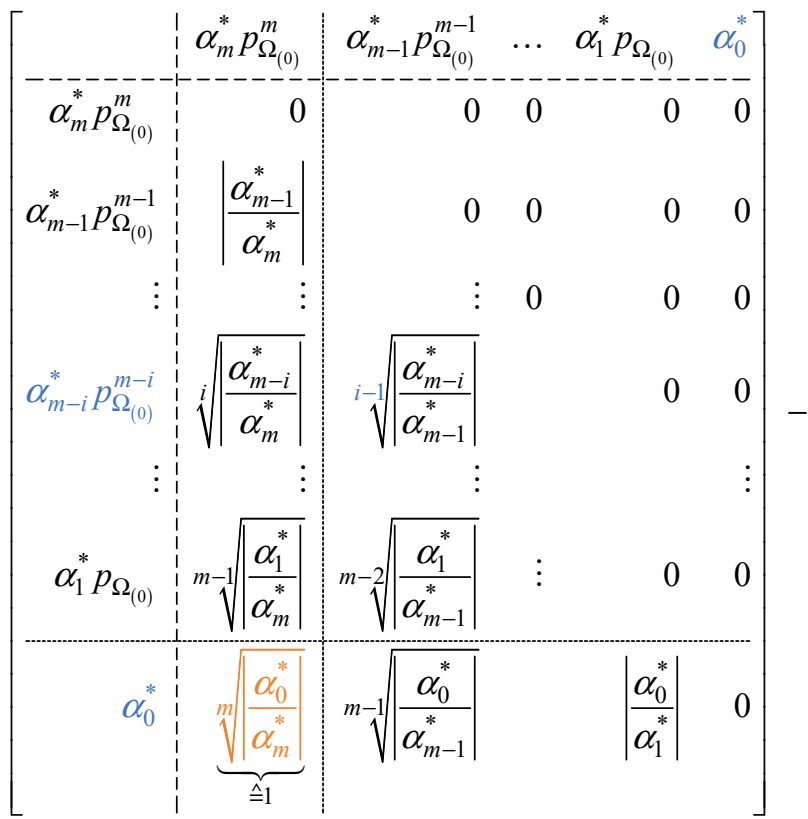

the matrix of the radii scales of enclosed invariants $\left(\alpha_{m-j}(t) p^{m-j} \ldots \alpha_{i}(t) p^{i}\right)$ is presented relatively to $\left(\alpha_{m}(t) p^{m}+\alpha_{0}(t)\right)=0$.

MATLAB $\mathbf{P}$-matrices filling procedure is presented below: 
function pmatrix=buildpmatrix (den)

$[\mathrm{m}, \mathrm{n}]=\mathrm{size}(\operatorname{den})$;

pmatrix=zeros $(n+1)$;

den_fliplr=fliplr(den);

for $i=1: n$

for $j=0: n-i$

pmatrix $(j+1+i, j+1)=n t h r o o t(a b s$ (den_fli $\operatorname{plr}(n+1-j-i) / \operatorname{den} f l i p l r(n+1-j)), i)$;

end end

Theorem. The nonstationary dynamic system with the transfer function

$W(p, t)=\frac{A(p, t)}{B(p, t)}=k_{0} \frac{\sum_{i=0}^{m} \alpha_{m-i}(t) p^{m-i}}{\sum_{j=0}^{n} \beta_{n-j}(t) p^{n-j}}=$

$=k_{0} \frac{\alpha_{m}(t) p^{m}+\alpha_{m-1}(t) p^{m-1}+\ldots+\alpha_{0}(t)}{\beta_{n}(t) p^{n}+\beta_{n-1}(t) p^{n-1}+\ldots+\beta_{0}(t)}$,

is stable at each time quantum $t_{k}$ if the component $\mathbf{P}_{i j}$ of each subdiagonal $2 \times(n-2)$ pieces of the main and secondary diagonals, of each row $n-2$ pieces and each column $n-1$ of the denominator polynomial $B(p, t) \mathbf{P}$ - matrix is under the lack of growth, as well as the same component $\mathbf{P}_{i j}$ of each superdiagonal $n-3$ pieces of her side diagonal, calculated at the same time quantum $t_{k}$ is under decreasing (11).

$$
\begin{aligned}
& \mathbf{P}_{B\left(p, t_{k}\right)}=
\end{aligned}
$$

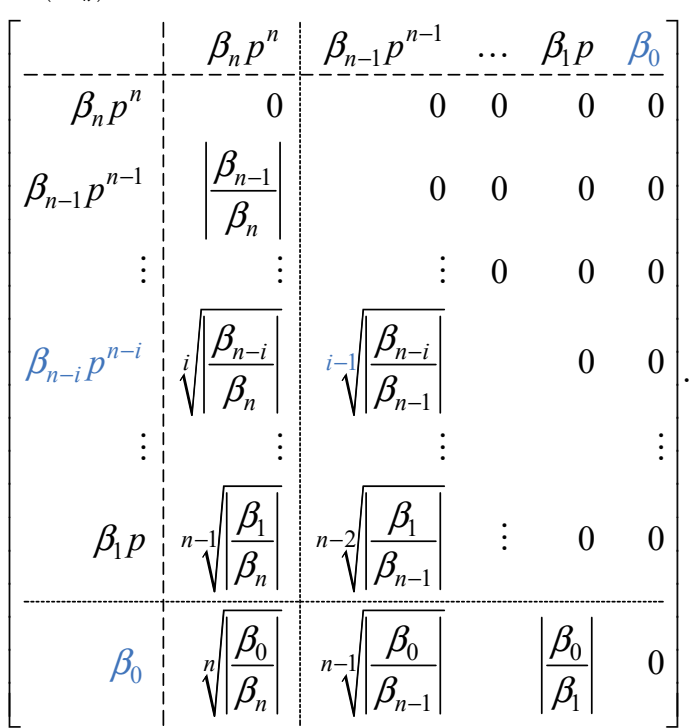

Each element $\mathbf{P}_{i j}$ of the lower triangular matrix $\mathbf{P}_{B\left(p, t_{k}\right)}$ is a linear dynamic system stability marker in the graphical interpretation of the adjacent and neighbouring $\mathbf{P}_{i j \pm 1}$ relationship (Fig. 2).

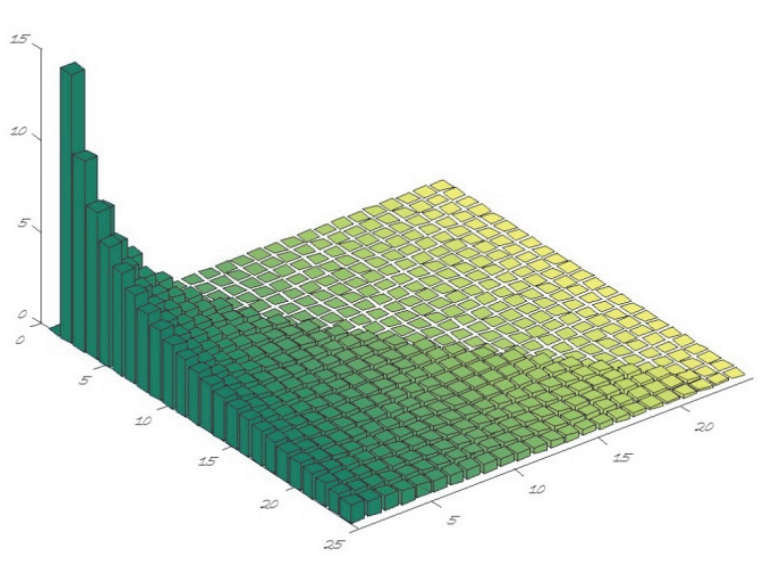

Fig. 2. Probability to generate this harmony in $\mathbf{P}$-matrix of the solutions space with a selection of random samples in the primary space - is equal to 0 !

The columns reciprocal geometry, but not their absolute values, is the essential event. The mentioned facts in a formulaic interpretation correspond to:

$\left|\frac{\beta_{n-1}}{\beta_{n}}\right|>\left|\frac{\beta_{n-2}}{\beta_{n-1}}\right|>\ldots>\left|\frac{\beta_{n-j-1}}{\beta_{n-j}}\right|>\ldots>\left|\frac{\beta_{0}}{\beta_{1}}\right|$ for the first,

$\ldots \sqrt[i]{\left|\frac{\beta_{n-i} \mid}{\beta_{n}}\right|}>\sqrt[i]{\left|\frac{\beta_{n-1-i}}{\beta_{n-1}}\right|}>\ldots>\sqrt[i]{\left|\frac{\beta_{n-j-i}}{\beta_{n-j}}\right|}>\ldots>\sqrt[i]{\left|\frac{\beta_{0}}{\beta_{i}}\right|}$,

for the $i$-th

$\ldots \sqrt[n-1]{\left|\frac{\beta_{1}}{\beta_{n}}\right|}>\sqrt[n-1]{\left|\frac{\beta_{0}}{\beta_{n-1}}\right|}$ and for the last but one

subdiagonals of the main diagonal.

The same phenomenon occurs in component $\mathbf{P}_{i j}$ of each subdiagonal $2 \times(n-2)$ pieces of the main and secondary diagonals, of each row $n-2$ pieces and each column $n-1$ of the denominator polynomial $B(p, t) \quad \mathbf{P}$ - matrix is under the lack of growth, as well as the same component $\mathbf{P}_{i j}$ of each superdiagonal $n-3$ pieces of her side diagonal (Fig. 3-7):

$\sqrt{\left|\frac{\beta_{n-2}}{\beta_{n}}\right|}>\left|\frac{\beta_{n-2}}{\beta_{n-1}}\right|$ for the third,

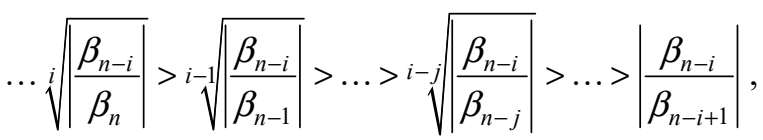

for the $i$-th

$\ldots \sqrt[n]{\mid \frac{\beta_{0}}{\beta_{n} \mid}}>\sqrt[n-1]{\mid \frac{\beta_{0}}{\beta_{n-1} \mid}}>\ldots>\sqrt[n-j]{\left|\frac{\beta_{0}}{\beta_{n-j}}\right|}>\ldots>\left|\frac{\beta_{0}}{\beta_{1}}\right|$ 
and for the $(n+1)$ rows, and

$\left|\frac{\beta_{n-1}}{\beta_{n}}\right|>\sqrt[2]{\left|\frac{\beta_{n-2}}{\beta_{n}}\right|}>\ldots>\sqrt[i]{\left|\frac{\beta_{n-i}}{\beta_{n}}\right|}>\ldots>\sqrt[n]{\left|\frac{\beta_{0}}{\beta_{n}}\right|}$ for the first column,

$$
\left|\frac{\beta_{n-j-1}}{\beta_{n-j}}\right|>\sqrt[2]{\left|\frac{\beta_{n-j-2}}{\beta_{n-j}}\right|}>\ldots>\sqrt[i-j]{\left|\frac{\beta_{n-i}}{\beta_{n-j}}\right|}>\ldots>\sqrt[n-j]{\left|\frac{\beta_{0}}{\beta_{n-j}}\right|},
$$

$\ldots\left|\frac{\beta_{1}}{\beta_{2}}\right|>\sqrt{\left|\frac{\beta_{0}}{\beta_{2}}\right|}$ and the $(n-1)$ columns, and also

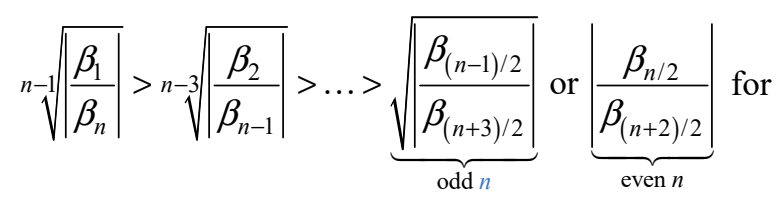

the first,

$$
\sqrt[i]{\left|\frac{\beta_{n-i}}{\beta_{n}}\right|}>\sqrt[i-2 \mid]{\left|\frac{\beta_{n-i+1}}{\beta_{n-1}}\right|}>\ldots>\underbrace{\sqrt{\left|\frac{\beta_{n-i / 2-1}}{\beta_{n-i / 2+1}}\right|}}_{\text {even } i} \text { or } \underbrace{\left|\frac{\beta_{(2 n-i-1) / 2}}{\beta_{(2 n-i+1) / 2}}\right|}_{\text {odd } i},
$$

for the $i$-th $\sqrt{\left|\frac{\beta_{n-3}}{\beta_{n}}\right|}>\left|\frac{\beta_{n-2}}{\beta_{n-1}}\right|$ and for the last but one

superdiagonals of the secondary diagonal, and

$\sqrt[n-1]{\mid \frac{\beta_{0}}{\beta_{n-1} \mid}}<\sqrt[n-3]{\mid \frac{\beta_{1}}{\beta_{n-2} \mid}}<\ldots<\underbrace{\sqrt{\left|\frac{\beta_{(n-3) / 2}}{\beta_{(n+1) / 2}}\right|}}_{\text {odd } n}$ or $\underbrace{\left|\frac{\beta_{n / 2-1}}{\beta_{n / 2}}\right|}_{\text {even } n}$ for

the first,

$\ldots \sqrt[n-j]{\mid \frac{\beta_{0}}{\beta_{n-j} \mid}}<\sqrt[n-j-2]{\mid \frac{\beta_{1}}{\beta_{n-j-1} \mid}}<\ldots<\underbrace{\sqrt{\mid \frac{\beta_{i}}{\beta_{i+2} \mid}}}_{\text {even } i}$ or $\underbrace{\mid \frac{\beta_{i}}{\beta_{i+1} \mid}}_{\text {odd } i}$, for $j$-th $\sqrt[3]{\left|\frac{\beta_{0}}{\beta_{3}}\right|}<\left|\frac{\beta_{1}}{\beta_{2}}\right|$ and for the last but one subdiagonals of the secondary diagonal.

The apparent awkwardness of the interdependent conditions, there are $2 n(n-1)$ inequations, demonstrates how much the balance of phase transition and getting to the cluster stability is delicate. Is there any probability to randomly generate a polynomial $\left(R_{i(\times)}: \beta_{n}^{*} p^{n}, \beta_{n-1}^{*} p^{n-1} \ldots \beta_{0}^{*}\right)$ ? That is a stable according to Hurwitz' criteria? This probability is equal to zero with the growth of the polynomial $B(p, t)$ order! It is similar to the probability of life appearance in the Universe. As, for example, can fifty-one randomly assigned component $\beta_{j}(t), \forall j=\overline{0, n}$ in $B(p, t)$ satisfy $2 * 50 * 49$ interdependent locations of the markers?

The violation of any one surely results the dynamic system in the dominant instability cluster. The limits/prohibitions plenty of the each marker $\mathbf{P}_{i j}$ location in the $\mathbf{P}$ matrix guarantees the adjustment mechanism existence of shallow and more profound system. The adjustment mechanism of shallow system is identified with the conditions of rows, columns and subdiagonals of the main diagonal, the adjustment mechanism of more profound system is defined with the subdiagonals conditions of its secondary diagonal.

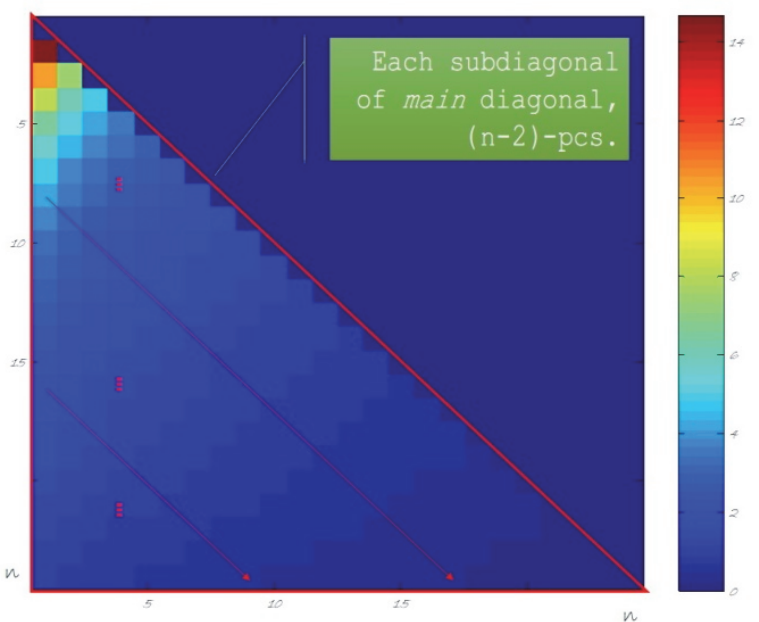

Fig. 3. About the lack of growth of the components $\mathbf{P}_{i j}$ of each subdiagonal of the lower triangular $\mathbf{P}_{B\left(p, t_{k}\right)}$ main diagonal.

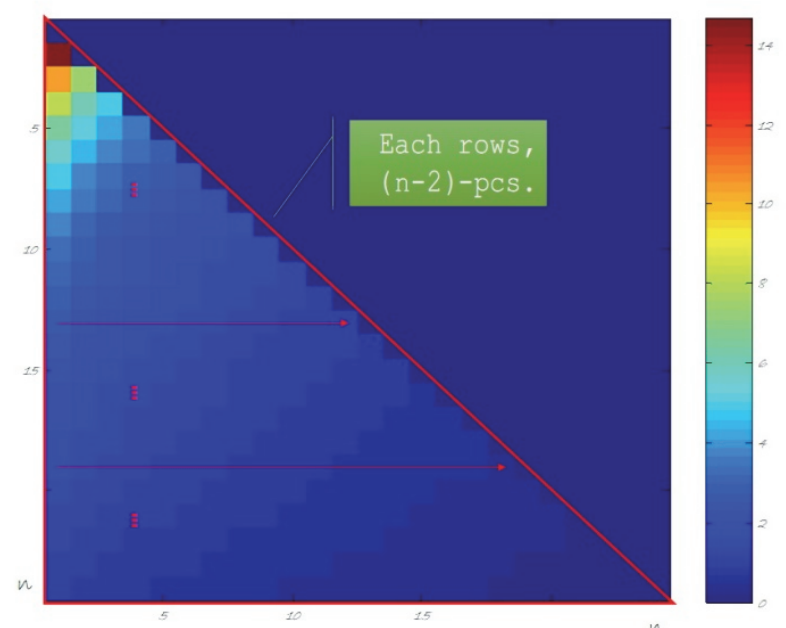

Fig. 4. About the lack of growth of the components $\mathbf{P}_{i j}$ of the lower triangular $\mathbf{P}_{B\left(p, t_{k}\right)}$ each row. 


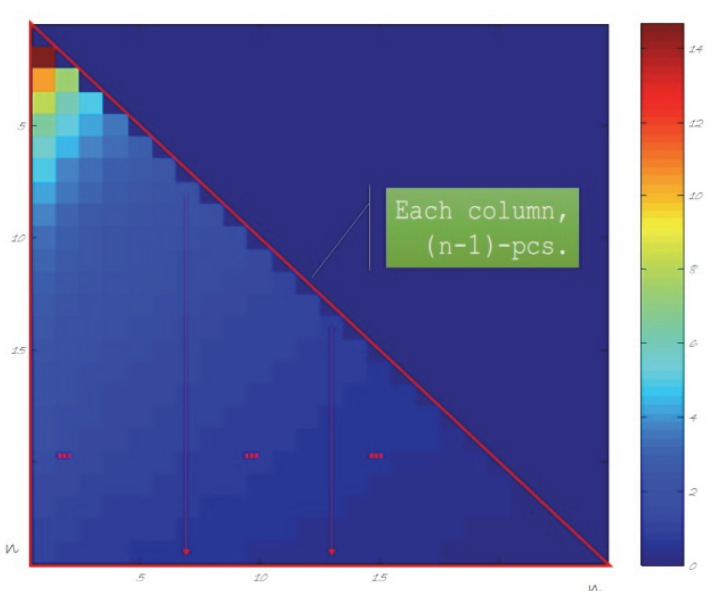

Fig. 5. About the lack of growth of the components $\mathbf{P}_{i j}$ of the lower triangular $\mathbf{P}_{B\left(p, t_{k}\right)}$ each column.

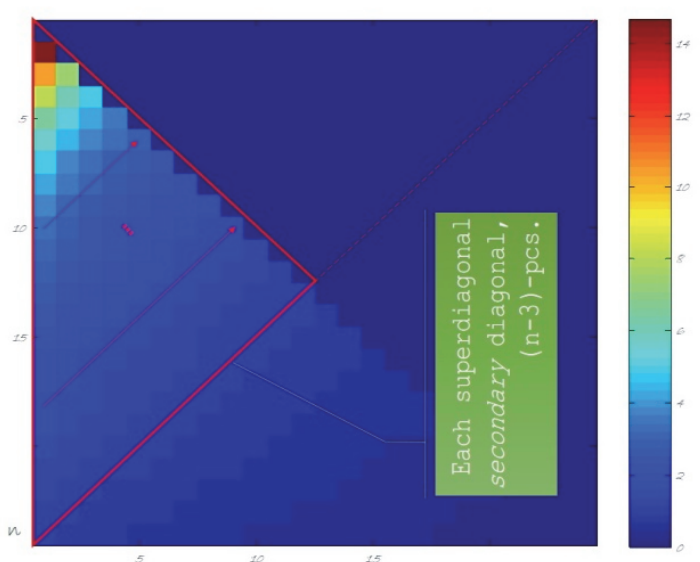

Fig. 6. About the non-decreasing of the components $\mathbf{P}_{i j}$ of each superdiagonal of the lower triangular $\mathbf{P}_{B\left(p, t_{k}\right)}$ secondary diagonal.

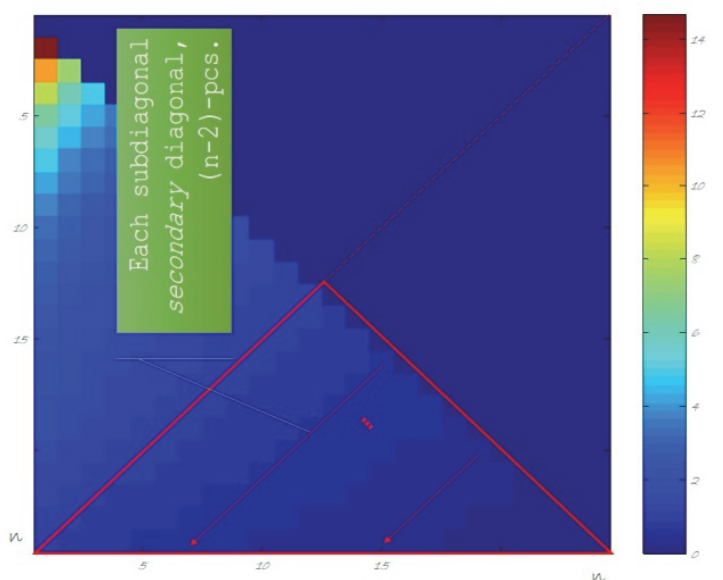

Fig. 7. About the lack of growth the components $\mathbf{P}_{i j}$ of each subdiagonal of the lower triangular $\mathbf{P}_{B\left(p, t_{k}\right)}$ secondary diagonal.
MATLAB procedure of check the columns, rows and subdiagonals lack of growth:

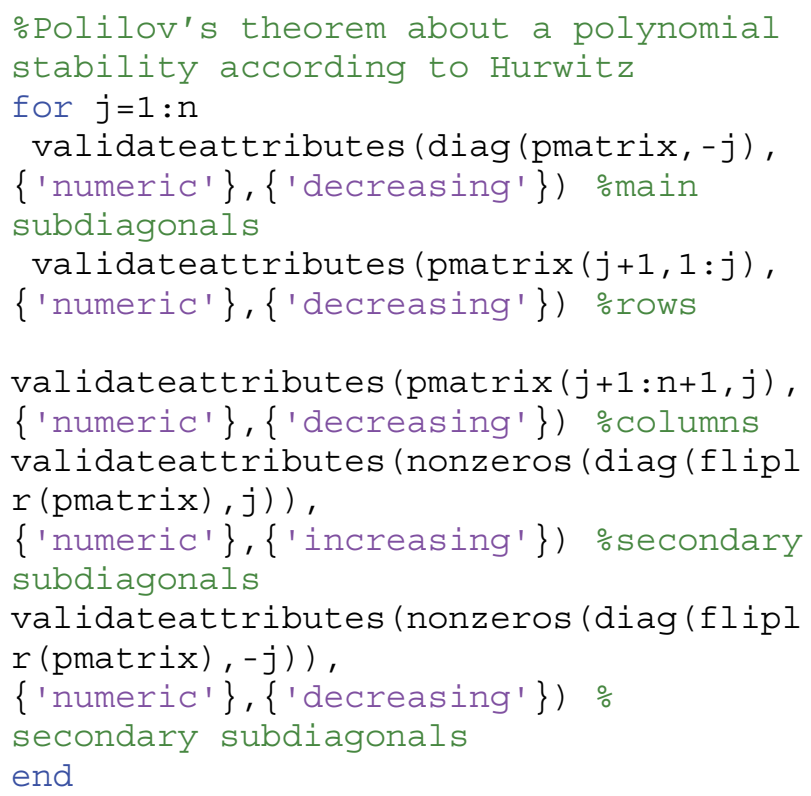

MATLAB procedure of plot 2D and 3D viewpoint of $\mathbf{P}$-matrix is presented here:
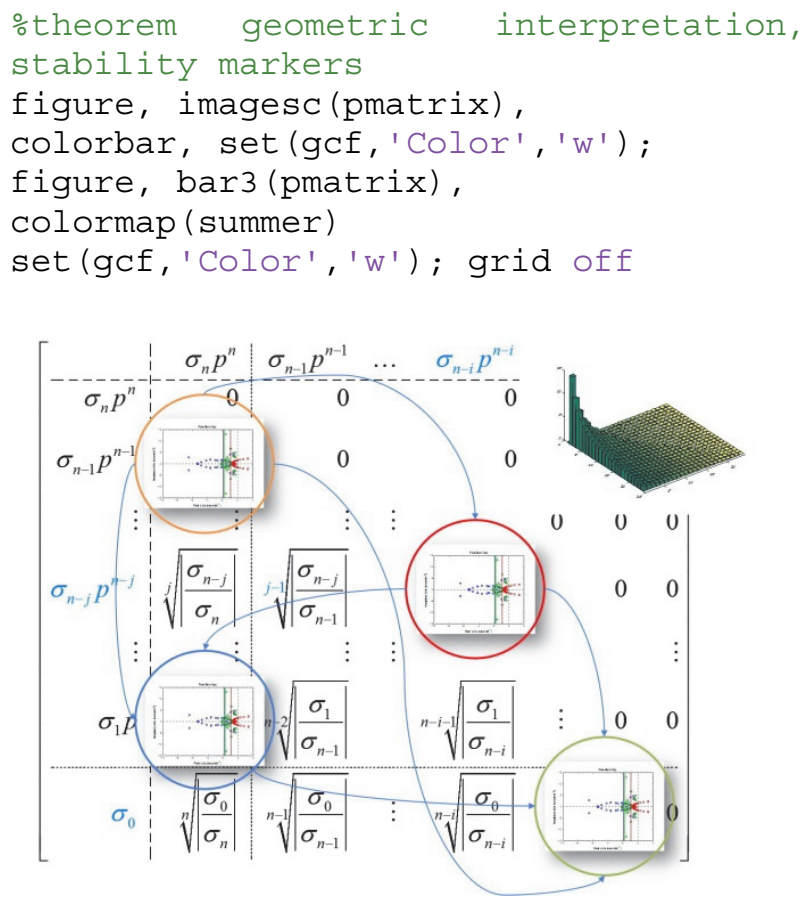

Fig. 8. There is a special radicals geometry in each cell of $\mathbf{P}$ matrix. Dominant of the neighboring components $\mathbf{P}_{i j \pm 1}$ allows to assign the stability markers.

\section{Conclusion}

The linear dynamic systems stability criterion, allowing one to identify the defective/failed $\sigma_{k}(t), \forall k \in \overline{0, n}$ from a set of non-stationary $\sigma_{j}(t), \forall j=\overline{0, n}$ in the characteristic polynomial $G(p, t)=0$ at each time 
quantum $t_{k}$ is presented for the first time. $\mathbf{P}$ - matrix, similar to the dispersive prism and the luminous flux, factorizes the dynamic system in the spectrum of geometric averages. The special order of the adjacent neighboring $\mathbf{P}_{i j \pm 1}$ alternation as in a strictly regulated sequence of the light spectrum color array shades is substantial and thus it allows assigning the stability markers.

Hurwitz' indicator of more than a hundred polynomials of the high orders with the radicals distribution $c_{j}(t), \forall j=\overline{1, n}$ according to Butterworth, Bessel, Graham- Lathrop etc., of various geometric averages $\Omega_{k}(t)=\underset{j=\overline{1, n}}{\operatorname{gmean}}\left(c_{j}(t)\right)$ has been geometrically confirmed. The nowlingly unstable dynamic systems, formed from Hurwitz' systems $G(p, t)=0$ with the intentional change of any component from $\sigma_{j}, \forall j \in \overline{0, n}$ that is refleëcted on Fig. 9, were being studied. In the $\mathbf{P}$ matrix the condition of the markers lack of growth of the corresponding subdiagonals, rows and columns, obviously these are some errors in $\beta_{15}$ and $\beta_{20}$, is disturbed.
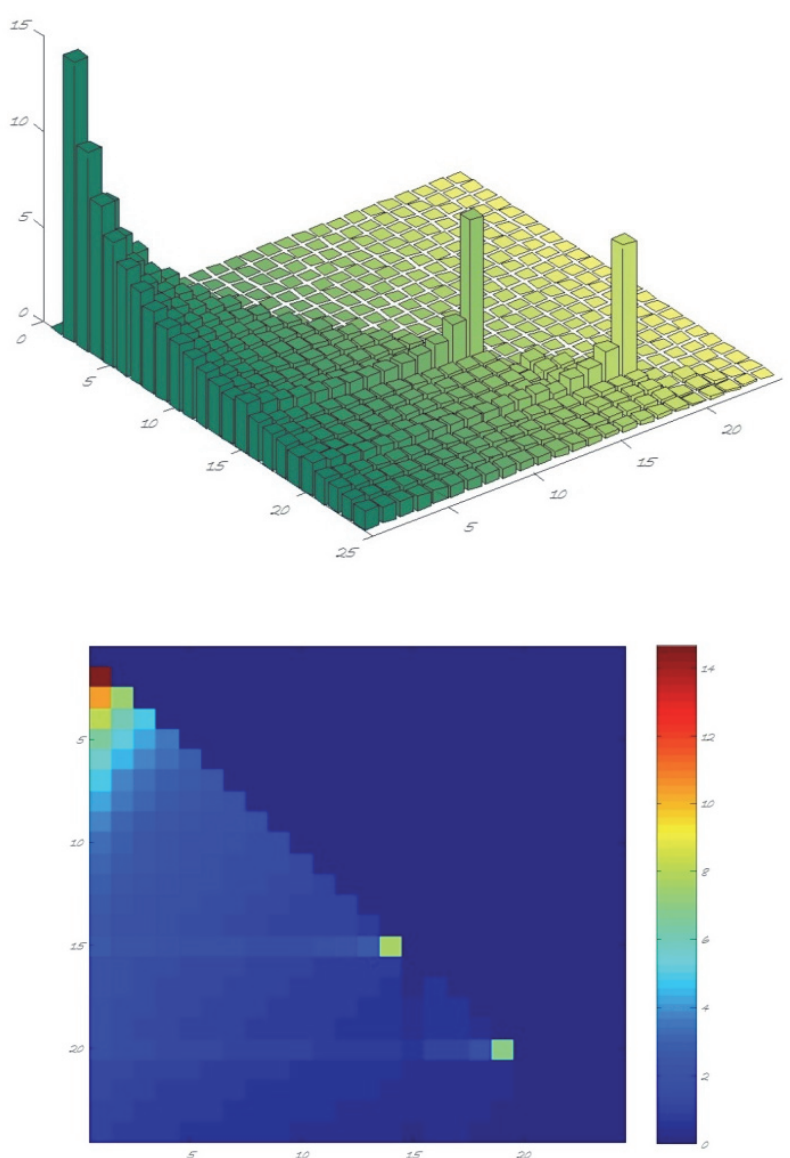

Fig. 9. A fault is in the solutions $\beta_{15} \times 15,0$ and $\beta_{20} \times 20,0$.

And, perhaps, the key moment, for what all this has been started is that it can be seen in $\mathbf{P}$ matrix how much the defective $\beta_{15}$ and $\beta_{20}$ have to be changed in order to fit them harmonically to the line with the adjacent "right" markers. The retention of non-stationary system in a very small stability cluster should be assigned on the adaptive computing potential, for example, on the mechanism of the artificial neural networks, which forcibly orders the markers $\mathbf{P}_{i j}$ in the $\mathbf{P}$ matrix at each time quantum $t_{k}$ in the current cycle and searchless criterion. A generation of the own Hurwitz' polynomials $G_{v}(p, t)=0$ in the modal control classical problems should be recognized as one of the niches of the $\mathbf{P}$ matrices effective application.

It is appropriate to speak about finding of the acceptable intervals $\left[\underline{\sigma}_{j} ; \quad \bar{\sigma}_{j}\right]$ of each $\sigma_{j}(t), \forall j=\overline{0, n}$ variation in the characteristic polynomial $G(p, t)=0$ with the $\mathbf{P}$ - matrices terms. It is a constructive alternative to the Kharitonov' theorem [7]. We can four corner polynomials, four $\mathbf{P}$ - matrices, markers, with the terms of interval polynomials:

$$
\begin{aligned}
& G_{1}(p)=\bar{\sigma}_{0}+\underline{\sigma}_{1} p+\underline{\sigma}_{2} p^{2}+\bar{\sigma}_{3} p^{3}+\bar{\sigma}_{4} p^{4}+\ldots, \\
& G_{2}(p)=\underline{\sigma}_{0}+\bar{\sigma}_{1} p+\bar{\sigma}_{2} p^{2}+\underline{\sigma}_{3} p^{3}+\underline{\sigma}_{4} p^{4}+\ldots, \\
& G_{3}(p)=\bar{\sigma}_{0}+\bar{\sigma}_{1} p+\underline{\sigma}_{2} p^{2}+\underline{\sigma}_{3} p^{3}+\bar{\sigma}_{4} p^{4}+\ldots, \\
& G_{4}(p)=\underline{\sigma}_{0}+\underline{\sigma}_{1} p+\bar{\sigma}_{2} p^{2}+\bar{\sigma}_{3} p^{3}+\underline{\sigma}_{4} p^{4}+\ldots
\end{aligned}
$$

\section{References}

1. M.M. Postnikov, Stable polynomials (Editorial URSS, Moscow, 2004)

2. Q.I. Rahman, G. Schmeisser, Analytic Theory of Polynomials: Critical Points, Zeros and Extremal Properties (Oxford: Clarendon Press, 2002)

3. N. Guglielmi, M. Manettac, Journal of Computational and Applied Mathematics, 292, 638-653 (2016)

4. N. Guglielmi, M. Gürbüzbalaban, M.L. Overton, SIAM J. Matrix Anal. Appl., 34(2), 709-737 (2013)

5. E.V. Polilov, Collection of Scientific Papers of Donbass State Technical University, 46, 102-121 (2016)

6. E.V. Polilov, Collection of scientific works "Bulletin of NTU "KhPI": The Problems of automated electric. Theory and Practice, 12(1121), 72-82 (2015)

7. V.L. Kharitonov, Diff. Eq., 1(11), 2086-2088 (1978) 